\title{
R\&D Competition between Commercial Open Source and Proprietary Software Firms
}

\author{
Mingqing Xing \\ School of Economics and Management, and Neural Decision Science Laboratory, \\ Weifang University, Weifang, Shandong, 261061, China \\ E-mail: mqxing1979@163.com
}

\begin{abstract}
Recent years, more and more firms develop commercial products based on open source software. The purpose of this study is to investigate $R \& D$ competition between commercial open source and proprietary software firms. This study constructs a mathematical model, and assumes that commercial open source software is inferior to its proprietary substitute in the usability and their functional qualities are the same before $R \& D$. It finds that: (i) proprietary software firm has higher incentive to improve the functional quality than commercial open source firm; (ii) when the $R \& D$ efficiency is low enough, the appearance of commercial open source software leads to the investment reduction of proprietary software firm. While when the $R \& D$ efficiency is high enough, the opposite may occur; (iii) from the viewpoint of social welfare, commercial open source firm invests too little, while proprietary software firm may invest too much.
\end{abstract}

Keywords: open source software, commercial open source software, proprietary software, $R \& D$, software function, software usability

\section{Introduction}

Since 1990s, the rapid development of open source software is an important phenomenon in the software industry. Unlike proprietary software, open source software (OSS) allows users to share source codes, identify and correct errors, and redistribute the software (for free, or at a charge) to other users [1]. MySQL (a database system), Linux (an operating system), StarOffice (an office suite) and Apache (a web server) are typical examples of open source software. Open source software can be categorized into community open source software and commercial open source software [2]. The former is owned by an open source community whose members do not get direct revenues from the software, and the latter is owned by a software vendor with propose of getting revenues from the software. In the study of Kumar et al [3], commercial open source software is defined as the privately built software product on the base of the publicly available source codes. Recent years more and more firms develop commercial products based on open source software. A typical example is Red Hat Inc, whose commercial versions of the free available Linux are designed to improve the usability and features of the Linux operating system.

In today's competitive market environment, proprietary software firms face many challenges, one of which comes from commercial open source software [4]. Due to the property of owners for commercial open source software and community open source software is different, this may lead to diverse strategic reactions when proprietary software firms compete with them. Moreover, commercial open source firms also need to know their R\&D strategies when they face competition from proprietary software. Compared with the traditional industries, the product update speed is faster in the software industry and consequently firms face stronger R\&D competition. Considering both community open source software and commercial open source software, this study 
examines $R \& D$ competition between commercial open source and proprietary software firms. A small share of the extant literature on the economics of open source studies R\&D investment. Verani [5] proposes a differentiated duopoly model, in which firms invest in the development of proprietary software or open source software. He finds that, for substitute software products (resp. for close to perfect complements), firms perform the most investment in software development if the software is open source (resp. proprietary); Economides and Katsamakas [6] compare the incentives of platform and application developers to invest in open source and proprietary operating systems. They find that the level of investment in applications is larger if the operating system is open source than if it is proprietary. The comparison of investment in operating systems depends on reputation effects and the number of developers; Xing [7] investigates the appearance of open source software on the R\&D risk in a market initially monopolized by proprietary firm, and finds that the R\&D risk level of proprietary firm is not higher in the market monopolized by proprietary software than in this market emerging open source software; Kumar et al [3] develop a two-sided model to examine R\&D competition between commercial open source firms. They find that a mandatory sharing setting may result in high-quality software products; Xing [8] compares innovation incentives for commercial open source firms under two typical licenses (the GNU General Public License (GPL) and the Berkeley Software Distribution License (BSD)), and finds that the innovation incentive towards software features (resp. usability) is always higher (resp. lower) under BSD than under GPL. Although the above research relates to both open source software and $R \& D$, they do not study $R \& D$ competition between commercial open source and proprietary software firms.

The rest of this paper is organized as follows. Section 2 gives the basic model. Section 3 solves the model and analyzes the equilibrium results. Section 4 compares the private optimum and the social optimum. The final section concludes this paper.

\section{The Model}

There are three types of software in a market: community open source software, commercial open source software and proprietary software. Community open source software is owned by a nonprofit open source community and it can be downloaded for free. Commercial open source software is provided by a commercial software firm who builds software products on the based of community open source software for revenues. Moreover, proprietary software is owned by a profit-maximizing proprietary firm.

Software quality depends on the usability and the functionality [9 11]. Software usability can be described by the ease of installation, learning and user interface, the efficiency of use, the level of technical support, etc. Software functionality can be measured by feature set, security, reliability, etc. Compared with proprietary software, community open source substitute lacks usability and it is generally less user-friendly. Commercial open source firms add value to community open source software by improving the functionality and/or the usability [3].

Empirical study shows that the user's technical ability is one of the key factors in open source adoption decisions [12]. As in the study of Choudhary and Zhou [9], this model assumes that consumers are heterogeneous in the level of technical skills, which is denoted by $\theta$. Since highly skilled consumers may not place as much emphasis on the usability as those less skilled consumers when they use a software product [4], this model assumes that a consumer's willingness to pay for the software usability decreases with his/her level of technical skills. The total number of consumers in the market is normalized to 1 and they are uniformly distributed over the interval $[0,1]$. Consequently $\theta$ is closed to 0 for highly skilled consumer and it is closed to 1 for lowly skilled consumer. 
The net utility for the generic consumer at $\theta \in[0,1]$ from using community open source software, commercial open source software and proprietary software are respectively defined as:

$$
u_{i}=\theta v_{i}+f_{i}-p_{i}
$$

In (1), $v_{i}, f_{i}$ and $p_{i}$ stand for the usability, functionality and price of software $i$ $(i=o, c, p)$ respectively. The subscripts ' $o$ ', ' $c$ ' and ' $p$ ' denote community open source software, commercial open source software and proprietary software respectively. Note that: (i) the price of community open source software is equal to zero (i.e., $p_{o}=0$ ) since it can be available from open source community for free; (ii) most of consumers prefer to use proprietary software that tends to be more user-friendly and better technical support. Consequently commercial open source software is assumed to be inferior to proprietary software in the usability (i.e., $v_{c}<v_{p}$ ). Moreover, commercial open source software is assumed to be better than community open source software in the usability (i.e., $v_{o}<v_{c}$ ).

Commercial open source firm builds software products based on community open source software. In order to increase the functional quality of software, commercial open source firm and proprietary firm engage in $R \& D$ activities. After R\&D investment, the functional qualities for commercial open source software and proprietary software are respectively:

$$
\begin{aligned}
& f_{c}=f_{o}^{0}+f_{1} \\
& f_{p}=f_{p}^{0}+f_{2}
\end{aligned}
$$

In (2) and (3), $f_{o}^{0}$ and $f_{p}^{0}$ denote the initial functional quality of community open source software and proprietary software respectively; $f_{1}$ and $f_{2}$ denote the R\&D effort for commercial open source firm and proprietary firm respectively.

Open source community does not conduct R\&D investment, but it can absorb part of the R\&D effort from commercial open source firm when its software codes open. Consequently, after R\&D the functional quality of community open source software is:

$$
f_{o}=f_{o}^{0}+\beta f_{1}
$$

In (4), $\beta$ denotes the proportion of open source community adopting the $R \& D$ effort of commercial open source firm. Note that: (i) $\beta$ may depend on the open source licenses. When commercial open source firms develop software product based on community open source software, they must comply with the corresponding open source licenses. They may be required to publicly release the software features in accordance with the terms of the open source license. Two typical open source licenses are the GNU General Public License (GPL) and the Berkeley Software Distribution license (BSD). Commercial open source firms must open the codes of software features under GPL, while it may not open under BSD [13]. Consequently, $\beta=0$ if a commercial open source firm keeps private the new developments made on the codes under BSD, and $\beta>0$ if open source community adopts some extensions of commercial open source firm under GPL; (ii) the initial functional qualities of community open source software and proprietary software are assumed to be equal (i.e., $f_{o}^{0}=f_{p}^{0}$ ).

The marginal consumer who is indifferent between using community open source software and commercial open source software (indexed by $\dot{\theta}$ ) is given by $u_{o}=u_{c}$, i.e.,

Solving the equation (5) for $\dot{\theta}$ yields:

$$
\hat{\theta} v_{o}+\left(f_{o}^{0}+\beta f_{1}\right)=\hat{\theta} v_{c}+\left(f_{o}^{0}+f_{1}\right)-p_{c}
$$

$$
\hat{\theta}=\frac{p_{c}-(1-\beta) f_{1}}{v_{c}-v_{o}}
$$


The marginal consumer who is indifferent between using commercial open source software and proprietary software (indexed by $\hat{\theta}$ ) is given by $u_{c}=u_{p}$, i.e.,

Solving the equation (7) for $\hat{\theta}$ gives:

$$
\grave{\theta} v_{c}+\left(f_{o}^{0}+f_{1}\right)-p_{c}=\grave{\theta} v_{p}+\left(f_{p}^{0}+f_{2}\right)-p_{p}
$$

$$
\hat{\theta}=\frac{p_{p}-p_{c}+f_{1}-f_{2}}{v_{p}-v_{c}}
$$

We assume that $\hat{\theta}$ and $\dot{\theta}$ meet $0<\hat{\theta}<\hat{\theta}<1$ and the market is full coverage. These assumptions can guarantee that the demands for three types of software are positive and the sum of them equals 1 . According to (6) and (8), the demand functions for open source community, commercial open source firm and proprietary firm are:

$$
\begin{gathered}
d_{o}=\hat{\theta}-0=\frac{p_{c}-(1-\beta) f_{1}}{v_{c o}} \\
d_{c}=\hat{\theta}-\hat{\theta}=\frac{f_{1}-f_{2}+p_{p}-p_{c}}{v_{p c}}-\frac{p_{c}-(1-\beta) f_{1}}{v_{c o}} \\
d_{p}=1-\hat{\theta}=1-\frac{f_{1}-f_{2}+p_{p}-p_{c}}{v_{p c}}
\end{gathered}
$$

In (9), (10) and (11), $v_{c o}=v_{c}-v_{o}$ and $v_{p c}=v_{p}-v_{c}$. Consequently, the profit functions for three types of software providers are given, respectively, by:

$$
\begin{gathered}
\pi_{o}=0 \\
\pi_{c}=p_{c} d_{c}-r f_{1}^{2}=p_{c}\left(\frac{f_{1}-f_{2}+p_{p}-p_{c}}{v_{p c}}-\frac{p_{c}-(1-\beta) f_{1}}{v_{c o}}\right)-r f_{1}^{2} \\
\pi_{p}=p_{p} d_{p}-r f_{2}^{2}=p_{p}\left(1-\frac{f_{1}-f_{2}+p_{p}-p_{c}}{v_{p c}}\right)-r f_{2}^{2}
\end{gathered}
$$

In (13) and (14), $r$ measures the R\&D efficiency, and the greater $r$ the lower the R\&D efficiency.

The timing of the model is as follows. Commercial open source firm and proprietary firm decide their R\&D effort in the first stage, and they set their software prices in the second stage.

\section{The Solution of the Model}

As usual, we solve the equilibrium results by backwards induction and start with a price competition stage. Commercial open source firm and proprietary firm pursue profit maximization. The first-order conditions with respect to $p_{c}$ and $p_{p}$ are:

$$
\begin{gathered}
\frac{\partial \pi_{c}}{\partial p_{c}}=\frac{f_{1}-f_{2}+p_{p}-2 p_{c}}{v_{p c}}-\frac{2 p_{c}-(1-\beta) f_{1}}{v_{c o}}=0 \\
\frac{\partial \pi_{p}}{\partial p_{p}}=1-\frac{f_{1}-f_{2}+2 p_{p}-p_{c}}{v_{p c}}=0
\end{gathered}
$$

Solving (15) and (16) gives equilibrium prices:

$$
\begin{gathered}
p_{c}=\frac{v_{c o}\left(v_{p c}+f_{1}-f_{2}\right)+2 v_{p c}(1-\beta) f_{1}}{4 v_{p o}-v_{c o}} \\
p_{p}=\frac{2 v_{p o} v_{p c}-\left(2 v_{p o}-v_{c o}\right)\left(f_{1}-f_{2}\right)+v_{p c}(1-\beta) f_{1}}{4 v_{p o}-v_{c o}}
\end{gathered}
$$


In (17) and (18), $v_{p o}=v_{p}-v_{o}$. Obviously, the second-order conditions are met (because $\frac{\partial^{2} \pi_{c}}{\partial p_{c}^{2}}=-\frac{2 v_{p o}}{v_{p c} v_{c o}}<0$ and $\frac{\partial^{2} \pi_{p}}{\partial p_{p}^{2}}=-\frac{2}{v_{p c}}<0$ ).

Substituting (17) and (18) into (13) and (14), the profit functions on variables $f_{1}$ and $f_{2}$ are obtained:

$$
\begin{gathered}
\pi_{c}=\frac{v_{p o}\left[2 v_{p c}(1-\beta) f_{1}+v_{c o}\left(v_{p c}+f_{1}-f_{2}\right)\right]^{2}}{v_{c o} v_{p c}\left(4 v_{p o}-v_{c o}\right)^{2}}-r f_{1}^{2} \\
\pi_{p}=\frac{\left[2 v_{p c} v_{p o}+v_{p c}(1-\beta) f_{1}-\left(2 v_{p o}-v_{c o}\right)\left(f_{1}-f_{2}\right)\right]^{2}}{v_{p c}\left(4 v_{p o}-v_{c o}\right)^{2}}-r f_{2}^{2}
\end{gathered}
$$

Now we consider the R\&D competition stage. Commercial open source firm and proprietary firm choose $f_{1}$ and $f_{2}$ respectively to maximize their profit. The first-order conditions can be written as:

$$
\begin{aligned}
& \frac{\partial \pi_{c}}{\partial f_{1}}=\frac{2 v_{p o}\left[2 v_{p c}(1-\beta)+v_{c o}\right]\left[2 v_{p c}(1-\beta) f_{1}+v_{c o}\left(v_{p c}+f_{1}-f_{2}\right)\right]}{v_{c o} v_{p c}\left(4 v_{p o}-v_{c o}\right)^{2}}-2 r f_{1}=0 \\
& \frac{\partial \pi_{p}}{\partial f_{2}}=\frac{2\left(2 v_{p o}-v_{c o}\right)\left[2 v_{p c} v_{p o}+v_{p c}(1-\beta) f_{1}-\left(2 v_{p o}-v_{c o}\right)\left(f_{1}-f_{2}\right)\right]}{v_{p c}\left(4 v_{p o}-v_{c o}\right)^{2}}-2 r f_{2}=0
\end{aligned}
$$

The resulting equilibrium $\mathrm{R} \& \mathrm{D}$ efforts are:

$$
\begin{gathered}
f_{1}^{*}=\frac{v_{p o} v_{c o} v_{p c}\left(\begin{array}{c}
{\left[2 v_{p c}(1-\beta)+v_{c o}\right]\left[r v_{p c}\left(4 v_{p o}-v_{c o}\right)^{2}-\left(2 v_{p o}-v_{c o}\right)^{2}\right]} \\
-2 v_{p o}\left(2 v_{p o}-v_{c o}\right)\left[2 v_{p c}(1-\beta)+v_{c o}\right]
\end{array}\right)}{\left(\begin{array}{c}
\left\{r v_{c o} v_{p c}\left(4 v_{p o}-v_{c o}\right)^{2}-v_{p o}\left[2 v_{p c}(1-\beta)+v_{c o}\right]^{2}\right\}\left[r v_{p c}\left(4 v_{p o}-v_{c o}\right)^{2}-\left(2 v_{p o}-v_{c o}\right)^{2}\right] \\
+v_{p o} v_{c o}\left(2 v_{p o}-v_{c o}\right)\left[2 v_{p c}(1-\beta)+v_{c o}\right]\left[v_{p c}(1-\beta)-\left(2 v_{p o}-v_{c o}\right)\right]
\end{array}\right)} \\
f_{2}^{*}=\frac{v_{p c} v_{p o}\left(2 v_{p o}-v_{c o}\right)\left(\begin{array}{c}
2\left\{r v_{c o} v_{p c}\left(4 v_{p o}-v_{c o}\right)^{2}-v_{p o}\left[2 v_{p c}(1-\beta)+v_{c o}\right]^{2}\right\} \\
+v_{c o}\left[2 v_{p c}(1-\beta)+v_{c o}\right]\left[v_{p c}(1-\beta)-\left(2 v_{p o}-v_{c o}\right)\right]
\end{array}\right)}{\left(\begin{array}{c}
\left\{r v_{c o} v_{p c}\left(4 v_{p o}-v_{c o}\right)^{2}-v_{p o}\left[2 v_{p c}(1-\beta)+v_{c o}\right]^{2}\right\}\left[r v_{p c}\left(4 v_{p o}-v_{c o}\right)^{2}-\left(2 v_{p o}-v_{c o}\right)^{2}\right] \\
+v_{p o} v_{c o}\left(2 v_{p o}-v_{c o}\right)\left[2 v_{p c}(1-\beta)+v_{c o}\right]\left[v_{p c}(1-\beta)-\left(2 v_{p o}-v_{c o}\right)\right]
\end{array}\right)}
\end{gathered}
$$

The second-order conditions require:

$$
\begin{gathered}
\frac{\partial^{2} \pi_{c}}{\partial f_{1}^{2}}=\frac{2 v_{p o}\left[2 v_{p c}(1-\beta)+v_{c o}\right]^{2}}{v_{c o} v_{p c}\left(4 v_{p o}-v_{c o}\right)^{2}}-2 r<0 \\
\frac{\partial^{2} \pi_{p}}{\partial f_{2}^{2}}=\frac{2\left(2 v_{p o}-v_{c o}\right)^{2}}{v_{p c}\left(4 v_{p o}-v_{c o}\right)^{2}}-2 r<0
\end{gathered}
$$

Obviously, the above conditions are met if the parameters satisfy the following inequality:

$$
r>\max \left\{\frac{v_{p o}\left[2 v_{p c}(1-\beta)+v_{c o}\right]^{2}}{v_{c o} v_{p c}\left(4 v_{p o}-v_{c o}\right)^{2}}, \frac{\left(2 v_{p o}-v_{c o}\right)^{2}}{v_{p c}\left(4 v_{p o}-v_{c o}\right)^{2}}\right\}
$$

Analysis of the equilibrium R\&D efforts yields the following three propositions.

Proposition 1. $f_{1}^{*}<f_{2}^{*}$ (see Figure 1 and Figure 2).

The commercial open source firm's R\&D incentive is lower than that of proprietary firm. The reason is as follows. Commercial open source software's price is lower than proprietary software's price because commercial open source software is inferior to proprietary software in the usability. Consequently, commercial open source firm obtains less profit than proprietary firm, and it spends less money for R\&D investment. Note that this result depends on the assumption $f_{o}^{0}=f_{p}^{0}$ (i.e., the functional qualities of community open source software and proprietary software are the same before R\&D). If $f_{o}^{0}$ is much 
higher than $f_{p}^{0}$, the commercial open source firm's R\&D incentive may be higher than that of proprietary firm.

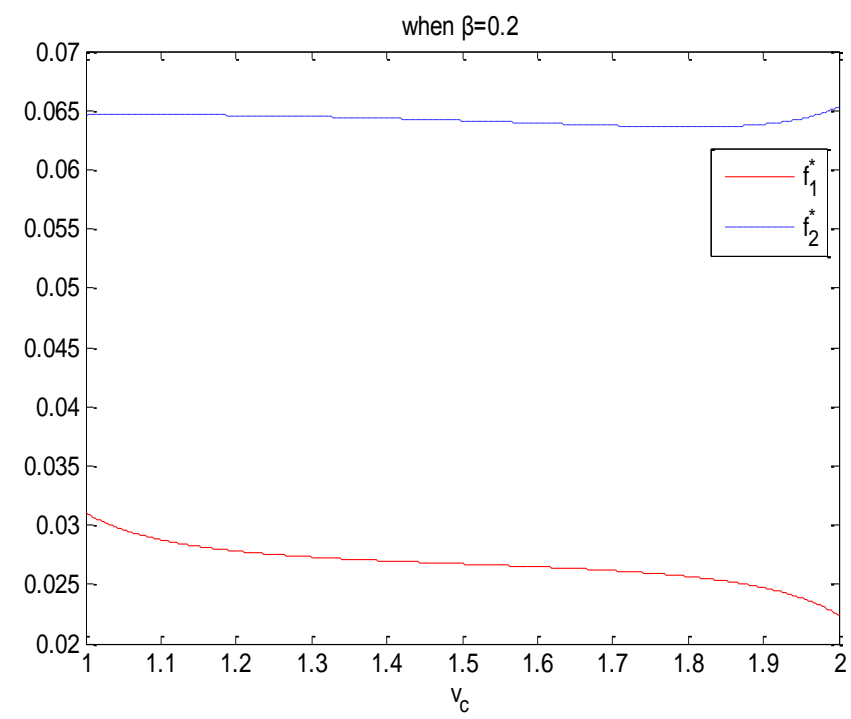

Figure 1. $f_{1}^{*}$ and $f_{2}^{*}$ when $\beta=0.2$ (given $v_{o}=0.8, v_{p}=2.2$ and $r=4$ )

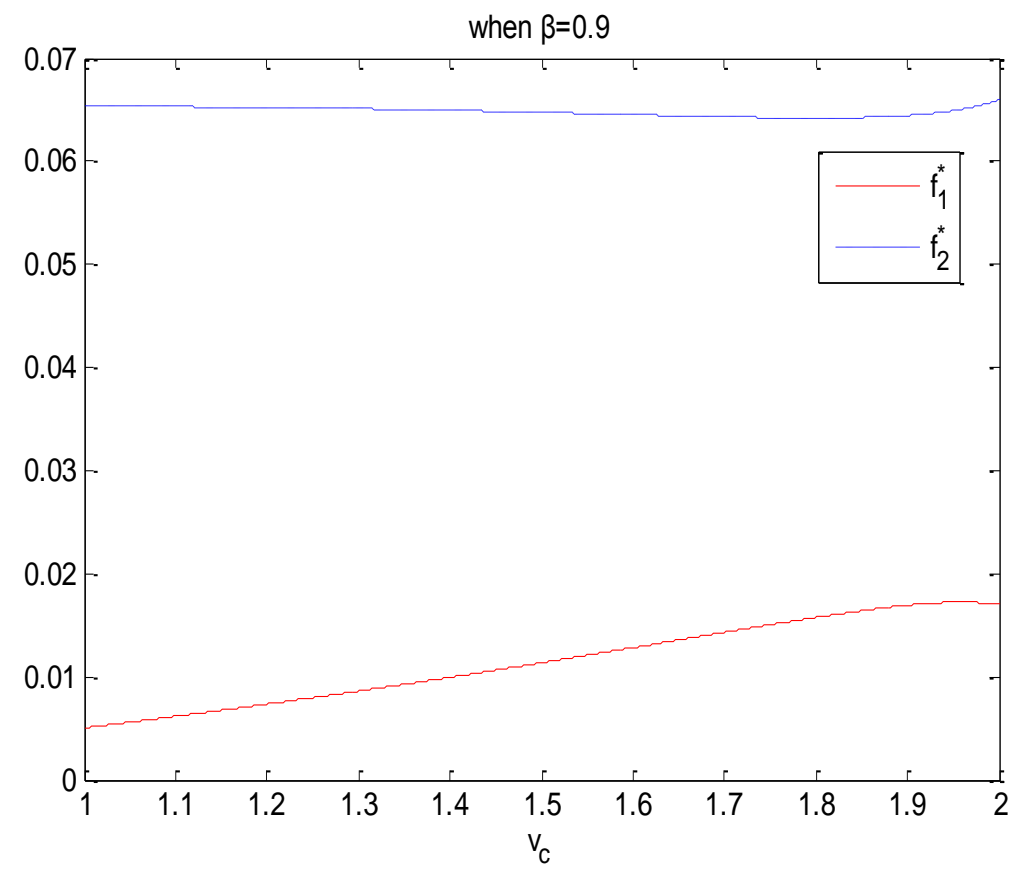

Figure 2. $f_{1}^{*}$ and $f_{2}^{*}$ when $\beta=0.9$ (given $v_{o}=0.8, v_{p}=2.2$ and $r=4$ )

Proposition 2. When $\beta$ is small enough, $f_{1}^{*}$ decreases with $v_{c}$; when $\beta$ is big enough, $f_{1}^{*}$ may increase with $v_{c}$ (see Figure 1 and Figure 2).

Proposition 2 gives the impact of $v_{c}$ on the commercial open source firm's R\&D incentive. The reason for this result is as follows. Increase of $v_{c}$ has two effects. On the one hand, it can increase the competitive advantage of commercial open source software relative to community open source software and can weaken competition between them. 
On the other hand, it can force price competition between commercial open source firm and proprietary firm. The smaller the open source community can absorb the R\&D effort of commercial open source firm $(\beta)$, the greater the former effect. The former effect is higher than the latter one if $\beta$ is small enough. This leads to the decrease of commercial open source firm's incentive to improve the functional quality. The opposite situation may appear if $\beta$ big enough.

Proposition 3. $f_{1}^{*}$ decreases with $\beta$ and $f_{2}^{*}$ increases with $\beta$ (see Figure 3).

Proposition 3 implies that, commercial open source (resp. proprietary) firm's R\&D investment decreases (resp. increases) as $\beta$ increases. The intuition behind this result is as follows. Increase of $\beta$ can decrease the competitive advantage of commercial open source software relative to community open source software. This can force competition between commercial open source software and community open source software, and can indirectly weaken competition between commercial open source software and proprietary software. Consequently, when $\beta$ increases, commercial open source firm has lower incentive to invest and proprietary firm has higher incentive to invest.

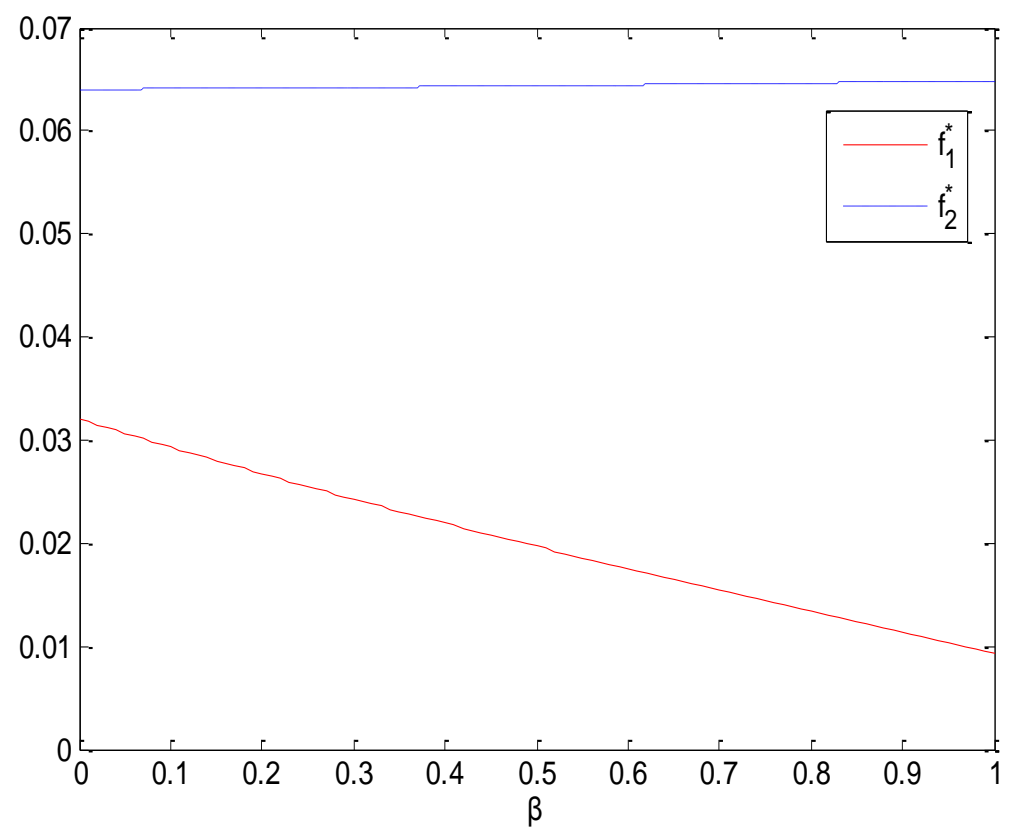

Figure 3. The Impact of $\beta$ on $f_{1}^{*}$ and $f_{2}^{*}$ (given $v_{o}=0.8, v_{c}=1.5, v_{p}=2.2$ and $r=4$ )

This part considers the case of proprietary software only facing competition from community open source substitute in order to analyze the commercial open source software's appearance on the proprietary software firm's R\&D incentive. In this case, the net utility for the generic consumer at $\theta \in[0,1]$ when he/she uses community open source software and proprietary software are respectively given by $u_{o}=\theta v_{o}+f_{o}^{0}$ and $u_{p}=\theta v_{p}+\left(f_{p}^{0}+f_{2}\right)-p_{p}$. We can prove that the equilibrium R\&D effort for proprietary firm is:

$$
f_{2}^{\#}=\frac{v_{p o}}{4 r v_{p o}-1}
$$


Comparing $f_{2}^{*}$ and $f_{2}^{\#}$, we obtain the following result.

Proposition 4. When $r$ is big, $f_{2}^{*}<f_{2}^{\#}$; when $r$ is small, $f_{2}^{*}<f_{2}^{\#}$ or $f_{2}^{*}>f_{2}^{\#}$ depends on $v_{c}$ and $\beta$ (see Figures 4 9).

Proposition 4 gives the impact of commercial open source competition on the proprietary firm's R\&D incentive. The reason for this result is as follows. When the R\&D efficiency is sufficiently low (i.e., $r$ is big enough), it is too expensive for proprietary firm to perform $R \& D$ and proprietary firm prefers to reduce $R \& D$ investment. Consequently, proprietary firm responds to competition from commercial open source software by decreasing R\&D investment when the R\&D efficiency is sufficiently low. Since the increase of commercial open source software's usability $\left(v_{c}\right)$ forces competition between commercial open source software and proprietary software, and the increase of $\beta$ weakens their competition indirectly, $f_{2}^{*}<f_{2}^{\#}$ or $f_{2}^{*}>f_{2}^{\#}$ may depend on $v_{c}$ and $\beta$ when the R\&D efficiency is small.

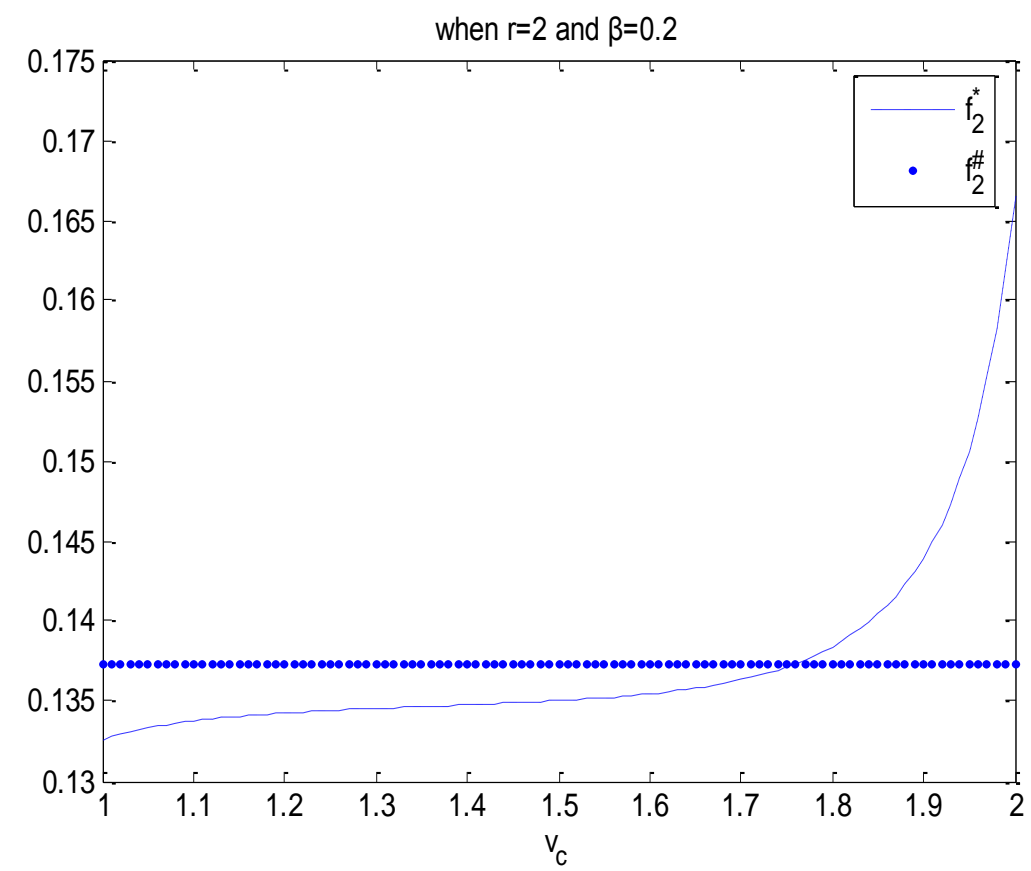

Figure 4. $f_{2}^{*}$ and $f_{2}^{\#}$ when $r=2$ and $\beta=0.2$ (given $v_{o}=0.8$ and $v_{p}=2.2$ ) 


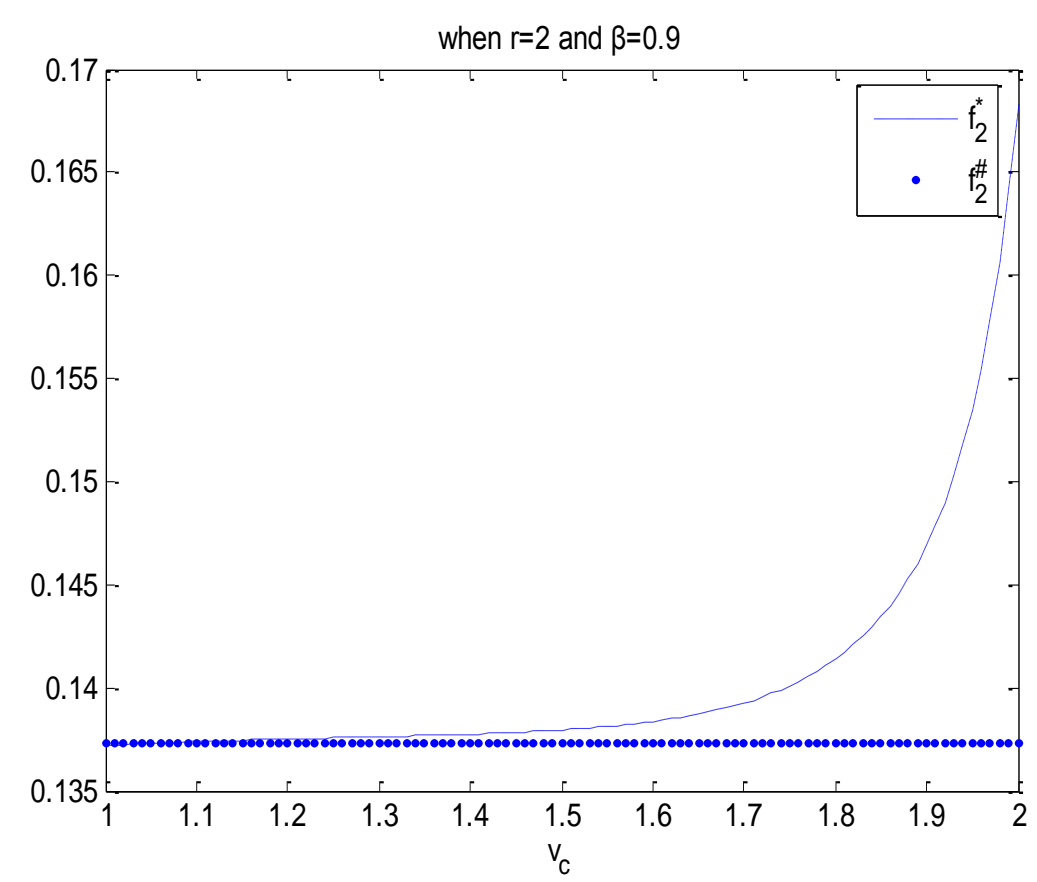

Figure 5. $f_{2}^{*}$ and $f_{2}^{\#}$ when $r=2$ and $\beta=0.9$ (given $v_{o}=0.8$ and $v_{p}=2.2$ )

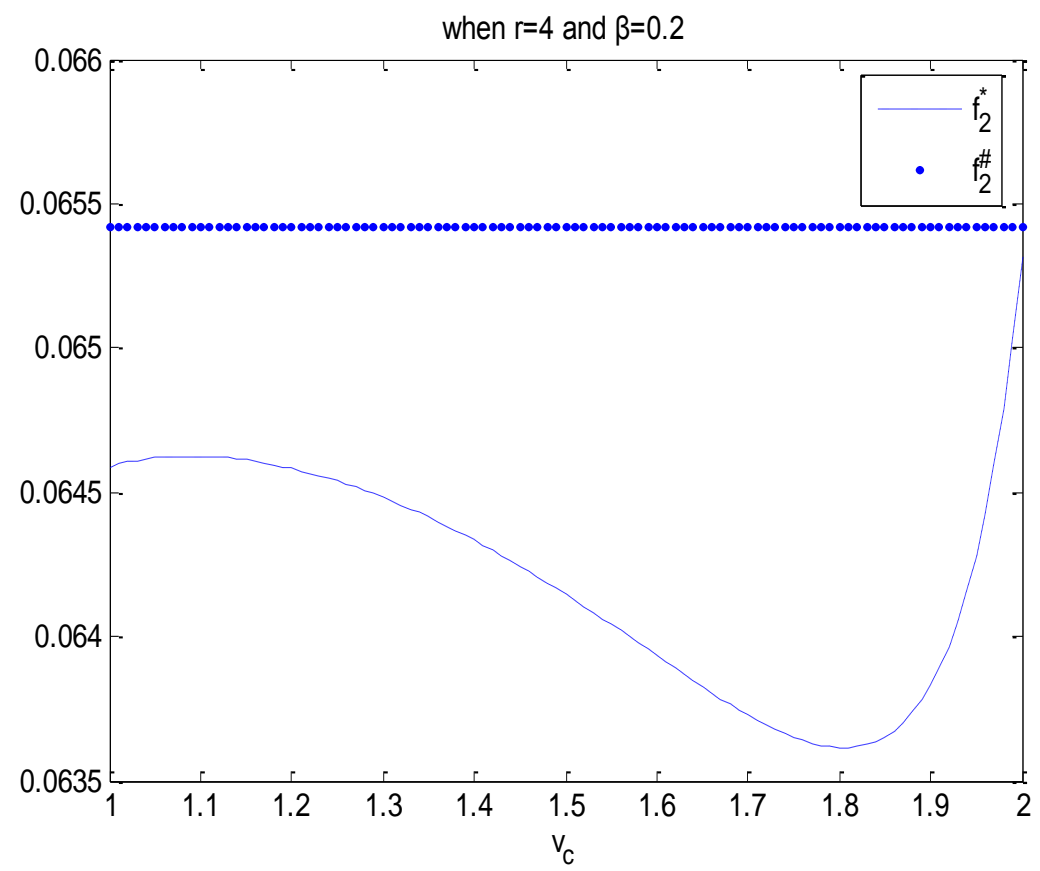

Figure 6. $f_{2}^{*}$ and $f_{2}^{\#}$ when $r=4$ and $\beta=0.2$ (given $v_{o}=0.8$ and $v_{p}=2.2$ ) 


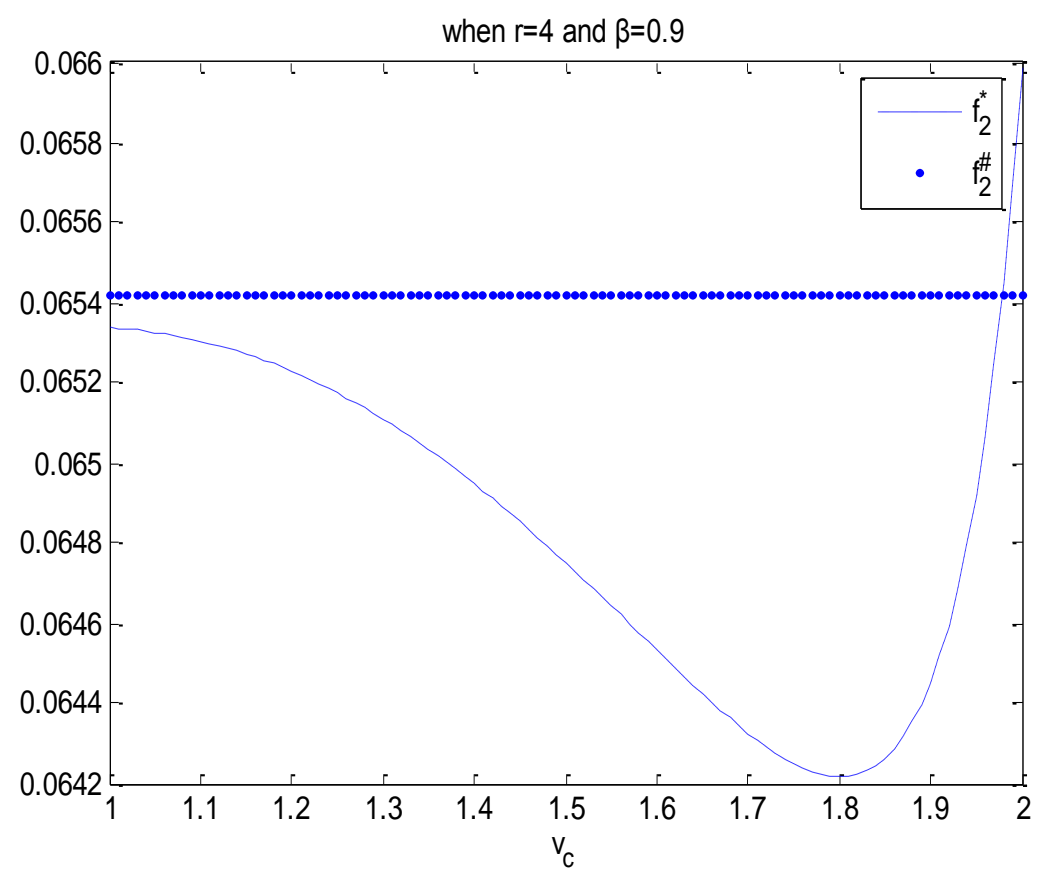

Figure 7. $f_{2}^{*}$ and $f_{2}^{\#}$ when $r=4$ and $\beta=0.9$ (given $v_{o}=0.8$ and $v_{p}=2.2$ )

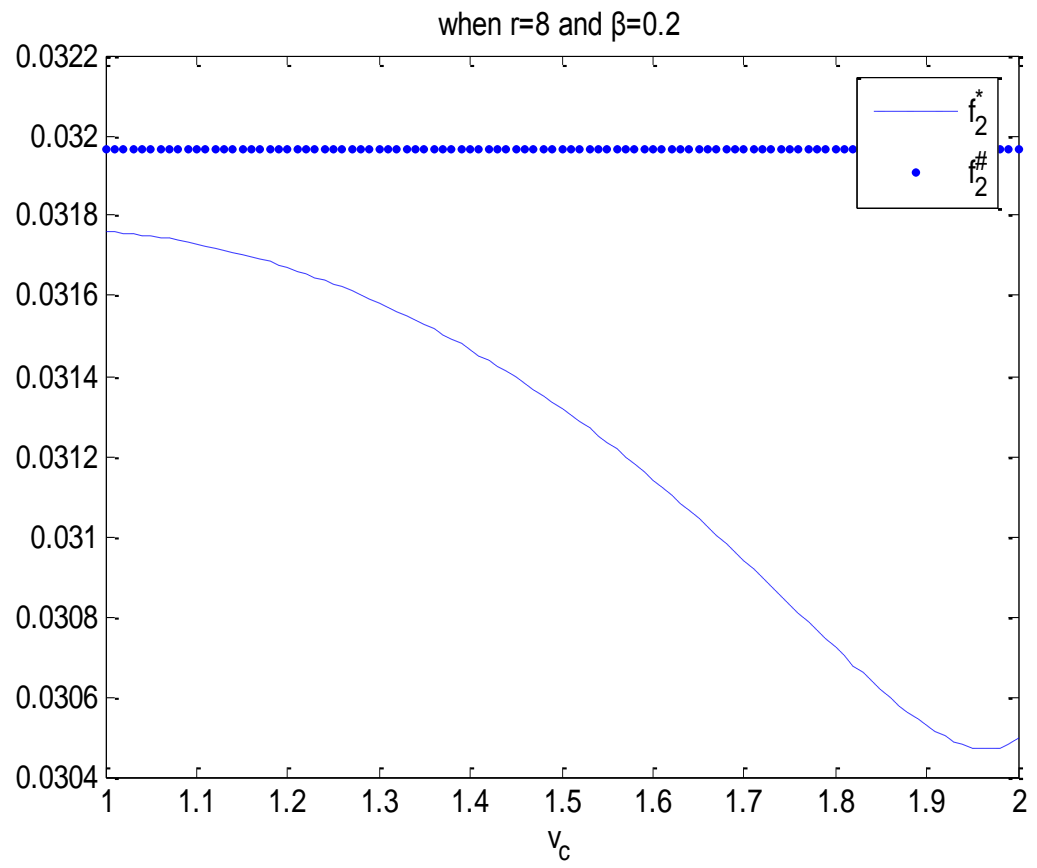

Figure 8. $f_{2}^{*}$ and $f_{2}^{\#}$ when $r=8$ and $\beta=0.2$ (given $v_{o}=0.8$ and $v_{p}=2.2$ ) 


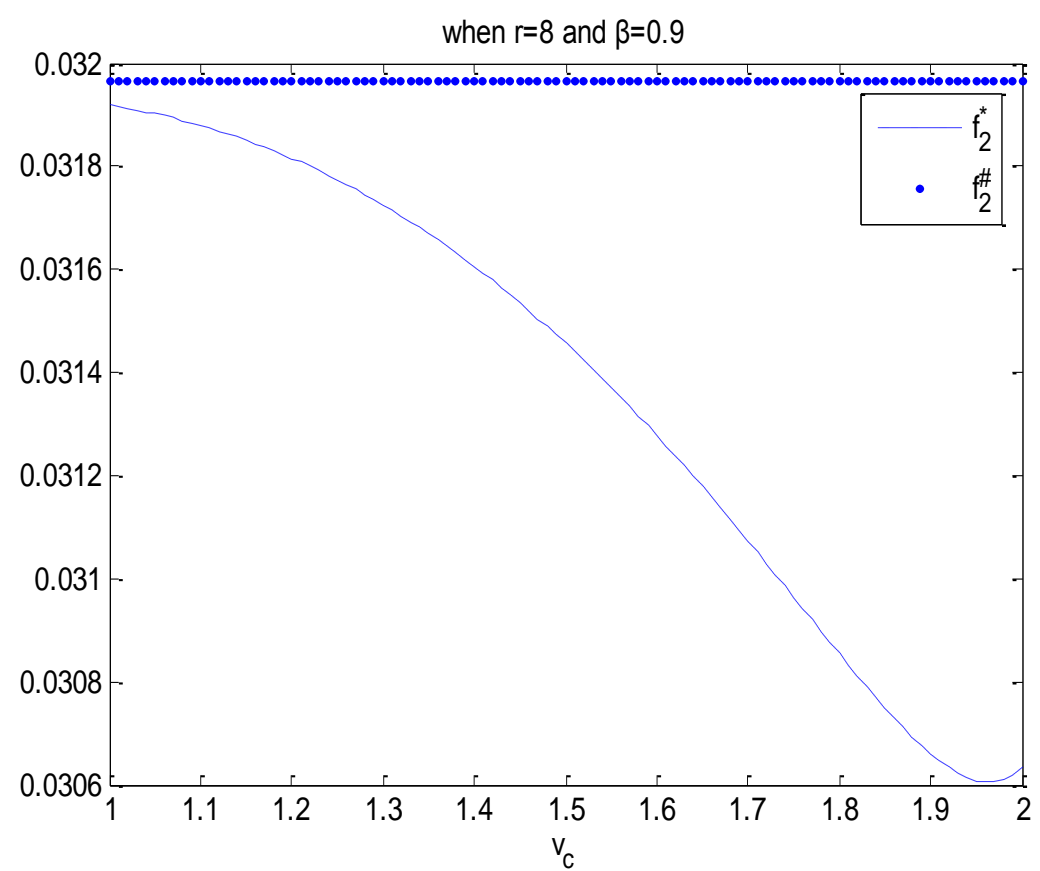

Figure 9. $f_{2}^{*}$ and $f_{2}^{\#}$ when $r=8$ and $\beta=0.9$ (given $v_{o}=0.8$ and $v_{p}=2.2$ )

\section{The Social Optimum}

We now proceed to analyze the optimal R\&D for a benevolent social planner whose objective is to maximize the value of social welfare. To this aim, consumer surplus can be computed as follows:

$$
C S=\int_{0}^{b}\left(\theta v_{o}+f_{o}^{0}+\beta f_{1}\right) d \theta+\int_{\theta}^{\grave{\theta}}\left(\theta v_{c}+f_{o}^{0}+f_{1}-p_{c}\right) d \theta+\int_{\theta}^{1}\left(\theta v_{p}+f_{p}^{0}+f_{2}-p_{p}\right) d \theta
$$

Social welfare is the sum of two firms' profits and consumer surplus, which is specified as follows:

$$
S W=\pi_{p}+\pi_{c}+C S=\int_{0}^{\vartheta}\left(\theta v_{o}+f_{o}^{0}+\beta f_{1}\right) d \theta+\int_{\theta}^{\vartheta}\left(\theta v_{c}+f_{o}^{0}+f_{1}\right) d \theta+\int_{\theta}^{1}\left(\theta v_{p}+f_{p}^{0}+f_{2}\right) d \theta-r f_{1}^{2}-r f_{2}^{2}
$$

We assume that the social planner only adjusts the R\&D investment, but the software price is determined by competition. Submitting (17) and (18) into (6) and (8) yields:

$$
\begin{gathered}
\hat{\theta}=\frac{\left(2 v_{p o}-v_{c o}\right)\left(v_{p c}+f_{1}-f_{2}\right)-v_{p c}(1-\beta) f_{1}}{v_{p c}\left(4 v_{p o}-v_{c o}\right)} \\
\hat{\theta}=\frac{v_{c o}\left(v_{p c}+f_{1}-f_{2}\right)-\left(2 v_{p o}+v_{c o}\right)(1-\beta) f_{1}}{v_{c o}\left(4 v_{p o}-v_{c o}\right)}
\end{gathered}
$$

Upon substitution of (31) and (32) in to (30), we obtain:

$$
S W=\frac{1}{2} v_{p}+f_{p}^{0}+f_{2}-\frac{\left(\begin{array}{c}
{\left[v_{c o}\left(v_{p c}+f_{1}-f_{2}\right)-\left(2 v_{p o}+v_{c o}\right)(1-\beta) f_{1}\right]\left[v_{c o}\left(v_{p c}+f_{1}-f_{2}\right)\right.} \\
\left.+3(1-\beta)\left(2 v_{p o}-v_{c o}\right) f_{1}\right]+\left[\left(2 v_{p o}-v_{c o}\right)\left(v_{p c}+f_{1}-f_{2}\right)\right. \\
\left.-v_{p c}(1-\beta) f_{1}\right]\left[\left(2 v_{p o}-v_{c o}\right) v_{p c}-(1-\beta) v_{p c} f_{1}+\left(6 v_{p o}-v_{c o}\right)\left(f_{2}-f_{1}\right)\right]
\end{array}\right)}{2 v_{c o}\left(4 v_{p o}-v_{c o}\right)^{2}}-r f_{1}^{2}-r f_{2}^{2}
$$

Differentiating (33) with respect to $f_{1}$ and $f_{2}$ respectively and setting them equal to zero, we can obtain the socially optimal R\&D efforts $f_{1}^{s}$ and $f_{2}^{s}$. Due to the expressions of $f_{1}^{s}$ and $f_{2}^{s}$ are very complicated, we do not give their specific expressions.

The second-order conditions require: 


$$
\begin{gathered}
\frac{\partial^{2} S W}{\partial f_{1}^{2}}=\frac{2\left(\begin{array}{c}
{\left[2 v_{p o}-\left(2 v_{p o}+v_{c o}\right) \beta\right]\left[v_{c o}+3(1-\beta)\left(2 v_{p o}\right.\right.} \\
\left.\left.-v_{c o}\right)\right]+\left(v_{p o}+\beta v_{p c}\right)\left[\left(7 v_{p o}-2 v_{c o}\right)-\beta v_{p c}\right]
\end{array}\right)}{2 v_{c o}\left(4 v_{p o}-v_{c o}\right)^{2}}-2 r<0 \\
\frac{\partial^{2} S W}{\partial f_{2}^{2}}=\frac{4 v_{p o}\left(3 v_{p o}-2 v_{c o}\right)}{v_{c o}\left(4 v_{p o}-v_{c o}\right)^{2}}-2 r<0
\end{gathered}
$$

Obviously, the above conditions are met if the parameters satisfy the following inequality:

$$
r>\max \left\{\frac{\left(\begin{array}{l}
{\left[2 v_{p o}-\left(2 v_{p o}+v_{c o}\right) \beta\right]\left[v_{c o}+3(1-\beta)\left(2 v_{p o}\right.\right.} \\
\left.\left.-v_{c o}\right)\right]+\left(v_{p o}+\beta v_{p c}\right)\left[7 v_{p o}-2 v_{c o}-\beta v_{p c}\right]
\end{array}\right)}{2 v_{c o}\left(4 v_{p o}-v_{c o}\right)^{2}}, \frac{2 v_{p o}\left(3 v_{p o}-2 v_{c o}\right)}{v_{c o}\left(4 v_{p o}-v_{c o}\right)^{2}}\right\}
$$

Now turn to evaluate social planning against the private optimum. By numerical analysis, we compare the size of $f_{i}^{*}$ and $f_{i}^{s}(i=1,2)$ (see Figure 7 and Figure 8).

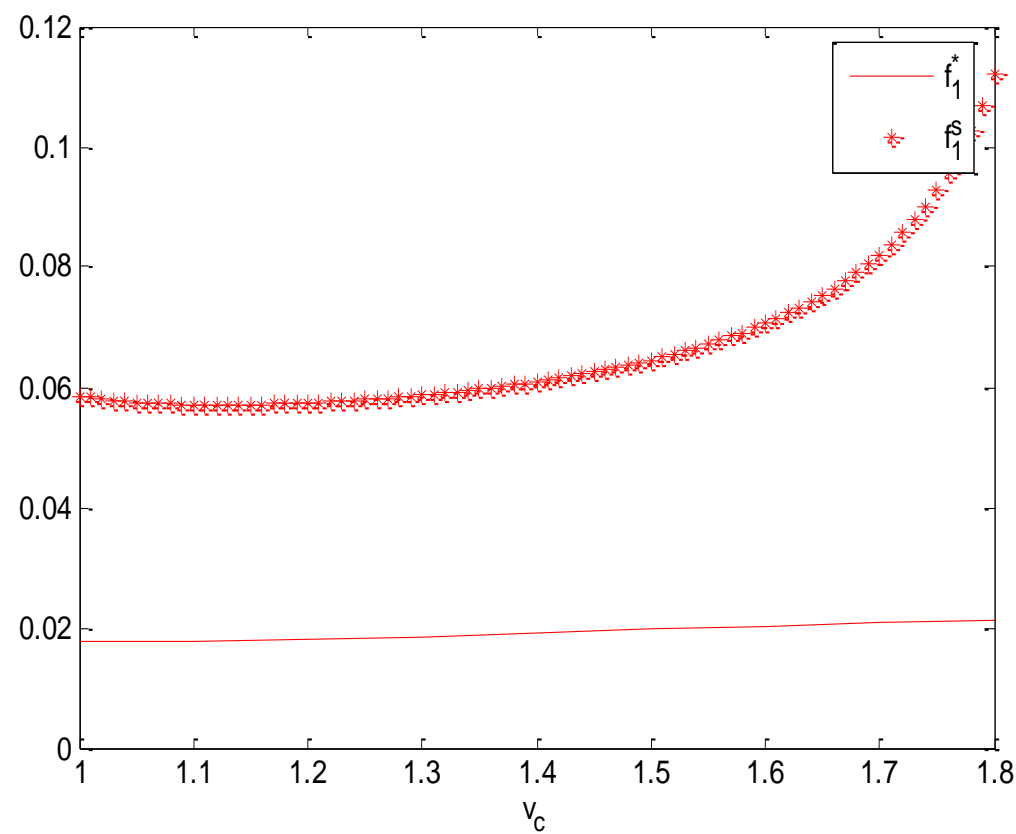

Figure 10. $f_{1}^{*}$ and $f_{1}^{s}$ (given $v_{o}=0.8, v_{p}=2.2, f_{o}^{0}=f_{p}^{0}=0.05, \beta=0.5$ and

$$
r=4 \text { ) }
$$




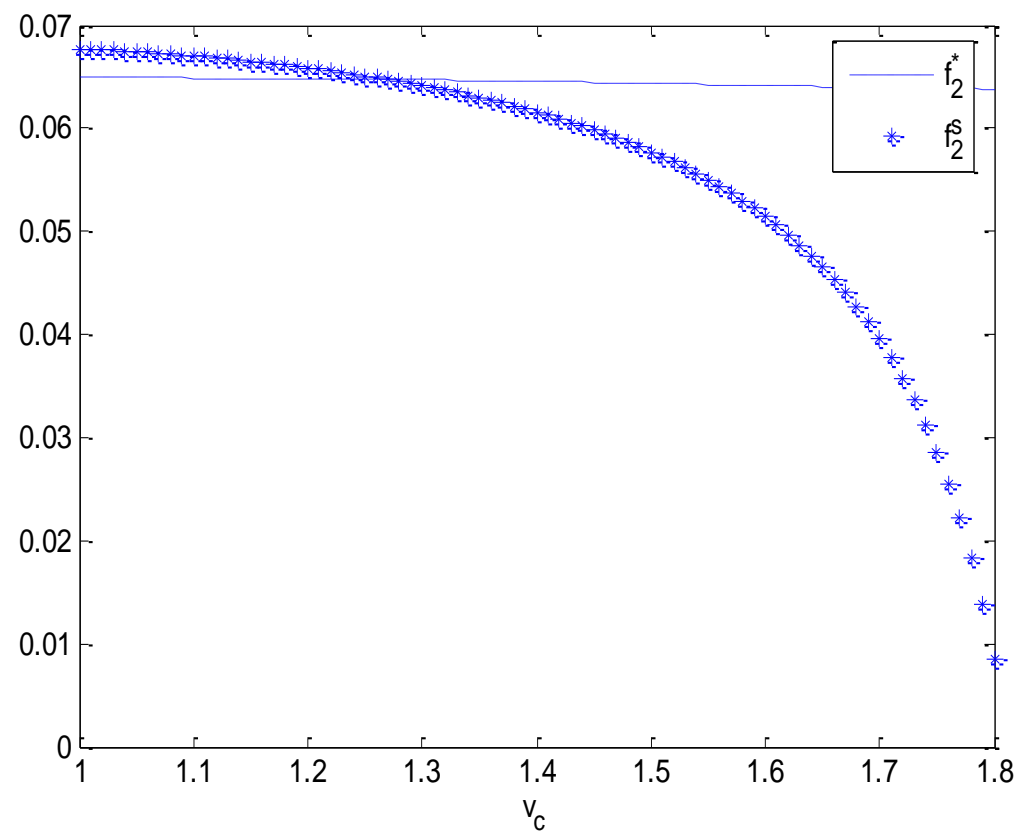

Figure 11. $f_{2}^{*}$ and $f_{2}^{s}$ (given $v_{o}=0.8, v_{p}=2.2, f_{o}^{0}=f_{p}^{0}=0.05, \beta=0.5$ and

$$
r=4 \text { ) }
$$

According to Figure 10 and Figure 11, we obtain the following conclusion.

Proposition 5. (i) $f_{1}^{*}<f_{1}^{s}$; (ii) when $v_{c}$ is small, $f_{2}^{*}<f_{2}^{s}$; when $v_{c}$ is big, $f_{2}^{*}>f_{2}^{s}$.

The above proposition demonstrates that, contrast to the social optimum, commercial open source firm invests too little in software functional quality. However, proprietary firm may invest too much if the commercial open source software's usability is close to the proprietary software's usability. Therefore, when the social planner develops software industry $\mathrm{R} \& \mathrm{D}$ policy, he/she necessarily distinguishes the types of software provider so as to make a correct public policy [14 17].

\section{Conclusions}

This paper examines R\&D competition between commercial open source firm and proprietary firm. Commercial open source firm builds software products on the basis of community open source software. Proprietary software is assumed to be better than commercial open source software in the usability and their functional quality is assumed to be the same before $R \& D$. This study mainly finds that: (i) proprietary firm invests more in the functional quality than commercial open source firm; (ii) when the R\&D efficiency is low, the appearance of commercial open source software leads to a decline in proprietary firm's investment; (iii) from the viewpoint of social welfare, commercial open source firm invests too little, while proprietary firm may invest too much.

\section{Acknowledgments}

The authors would like to thank anonymous referees for their valuable comments. We also acknowledge the financial support from the Shandong Institute of Humanities and Social Sciences Research Projects (No. J16WE27). 


\section{References}

[1] T. O'Reilly, "Lessons from Open-source Software Development", Communications of the ACM, vol. 42, no. 2, (1999), pp. 33-37.

[2] D. Riehle, "The Single-vendor Commercial Open Source Business Model", Information Systems and eBusiness Management, vol. 10, no. 1, (2012), pp. 5-17.

[3] V. Kumar, B. R. Gordon and K. Srinivasan, "Competitive Strategy for Open Source Software", Marketing Science, vol. 30, no. 6, (2011), pp. 1066-1078.

[4] R. Sen, "A Strategic Analysis of Competition between Open Source and Proprietary Software", Journal of Management Information Systems, vol. 24, no. 1, (2007), pp. 238-258.

[5] S. Verani, "Open Source Development in a Differentiated Duopoly", Working Paper 06-05, Available at: http://www.web.uwa.edu.au/_data/assets/pdf_file/0011/99236/06_05_Verani.pdf, (2006).

[6] N. Economides and E. Katsamakas, "Linux vs. Windows: a Comparison of Application and Platform Innovation Incentives for Open Source and Proprietary Software Platforms", NYU, Law and Economics Research Paper 05-21, Available at: http://ssrn.com/abstract=822894, (2005).

[7] M. Q. Xing, "The R\&D Risk for Proprietary Software Producer When Open Source Software Appears", Procedia Engineering, vol. 15, (2011), pp. 1382-1387.

[8] M. Q. Xing, "Comparative Study of Innovation Incentives between Commercial Open Source Software under Different Licenses", Research Journal of Applied Sciences, Engineering and Technology, vol. 5, no. 5, (2013), pp. 1633-1638.

[9] V. Choudhary and Z. Z. Zhou, "Impact of Competition from Open Source Software on Proprietary Software", Proceeding of the Institute for Operations Research and the Management Sciences Annual Meeting, Washington, America, (2007) November 4-7.

[10] M. Q. Xing, "The Impact of Commercial Open Source Software on Proprietary Software Producers and Social Welfare", Journal of Industrial Engineering and Management, vol. 7, no. 5, (2014), pp. 1183-1196.

[11] M. Q. Xing, "The Effect of Competition from Open Source Software on the Quality of Proprietary Software in the Presence of Network Externalities", Journal of Industrial Engineering and Management, vol. 8, no. 3, (2015), pp. 861-876.

[12] L. H. Lin, "Impact of User Skills and Network Effects on the Competition between Open Source and Proprietary Software", Electronic Commerce Research and Applications, vol. 7, no. 1, (2008), pp. 68-81.

[13] L. S. Laurent, "Understand Open Source and Free Software Licensing", O'Reilly Media, Cambridge, MA, (2004)

[14] M. Q. Xing, "On the Optimal Choices of R\&D Risk in a Market with Network Externalities”, Economic Modelling, vol. 38, (2014), pp. 71-74.

[15] M. Q. Xing, “The Game Analysis of Firms' R\&D Competition Strategies in Market with Network Externalities", China Social Sciences Press, Beijing, (2014).

[16] M. Q. Xing, "Research on Platform Competition Strategy from the Perspective of Two-sided Market Theory”, Enterprise Management publishing House, Beijing, (2016).

[17] M. L. Zheng, "Analysis on the Relationship between Industrial Structure Adjustment and Population Employment in Shandong Province", People's Publishing House, Beijing, (2015).

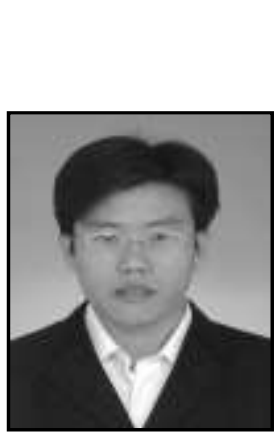

\section{Author}

Mingqing Xing, he is an associate professor in the school of economics and management at Weifang University, China. He received $\mathrm{PhD}$ in College of Science from China Agriculture University (2009). His current research interests mainly focus on software industry competition. His research is published in scientific journals such as "Economic Modelling" and "Journal of Industrial Engineering and Management". 